\title{
Formação em Odontologia e os estágios supervisionados em serviços públicos de saúde: percepções e vivências de estudantes
}

Talitha Rodrigues Ribeiro Fernandes Pessoa*; Ricardo Dias de Castro*; Claudia Helena Soares de Morais Freitas*; Altamira Pereira da Silva Reichert**; Franklin Delano Soares Forte*

* Doutor/a, Docente, Departamento de Clínica e Odontologia Social, Centro de Ciências da Saúde, Universidade Federal da Paraíba

** Doutora, Docente, Departamento de Enfermagem em Saúde Coletiva, Centro de Ciências da Saúde, Universidade Federal da Paraíba

Artigo originado do Trabalho de Conclusão de Curso de Talitha Rodrigues Ribeiro Fernandes Pessoa no Curso de Especialização em Ativação de Mudanças na formação Superior em Saúde, ENSP/FIOCRUZ

Recebido em 11/07/2017. Aprovado em 29/11/2017.

\begin{abstract}
RESUMO
O objetivo deste estudo foi compreender as percepções de estudantes de Odontologia de uma instituição pública de ensino superior sobre os estágios supervisionados em serviços públicos de saúde. Trata-se de uma pesquisa com abordagem qualitativa com o recurso de entrevistas semiestruturadas com 18 estudantes, selecionados por conveniência, matriculados do primeiro ao último ano do curso. As entrevistas, audiogravadas e transcritas, foram analisadas a partir da técnica de análise de conteúdo. Foram evidenciadas a aproximação com a população, a experiência profissional humanizada e o conhecimento construído pela vivência nos serviços, produzindo novas estratégias de atuação no Sistema Único de Saúde (SUS). As sugestões relacionaram-se à diversificação dos cenários de prática e a um melhor planejamento de atividades para o desenvolvimento de competências e habilidades. Por meio dos estágios, a formação volta-se para o SUS, que será novamente beneficiado com a inserção desses futuros profissionais na rede de serviços, com consequente melhoria do cuidado em saúde da população.
\end{abstract}

Descritores: Educação em Saúde. Serviços de Integração Docente-Assistencial. Educação em Odontologia. 


\section{INTRODUÇÃO}

A universidade tem o papel permanente de gerar saber para a sociedade, porém, a falta de articulação entre as instituições de ensino superior (IES) com as políticas públicas de saúde tem promovido a formação de profissionais descontextualizada com os princípios do Sistema Único de Saúde (SUS) ${ }^{1-4}$. A legislação que regulamenta o funcionamento do SUS elenca seu campo de atuação para a ordenação da formação de trabalhadores/profissionais de saúde, colaborando em atividades de ensino e pesquisas, mediante parcerias estabelecidas com as instituições de ensino ${ }^{4}$

Com a implantação das Diretrizes e Bases da Educação Nacional, em 1996, e das Diretrizes Curriculares Nacionais (DCN) para os cursos da saúde, a partir de 2001, muitos cursos foram incentivados para mudanças em suas matrizes curriculares ${ }^{5}$. Esse movimento visou à mudança no perfil do egresso, estimulando a diversificação dos cenários de aprendizagem, de forma a atender às demandas do SUS ${ }^{6}$.

As IES em saúde devem pensar na formação profissional voltada para os princípios e diretrizes do SUS, a partir da humanização das práticas em saúde, com a integração de conhecimentos gerais e específicos, habilidades teóricas e práticas, atitudes e valores éticos. Para isto, é necessário repensar a inserção desse profissional na sociedade e construir sua participação partindo do cotidiano, da reflexão e da formulação de propostas fundamentadas nos problemas enfrentados na rede de atenção à saúde ${ }^{7-12}$.

Dessa forma, o estágio supervisionado previsto nas DCN é uma atividade que tem contribuído para o aperfeiçoamento técnico bem como a compreensão de realidades sociais, culturais, econômicas e epidemiológicas, promovendo uma formação acadêmica baseada em contextos reais. As atividades devem ser realizadas com vistas à formação de um profissional sensível às necessidades da população, buscando a integração das ações de natureza preventiva e curativa, teoria e prática, e entre ensino, extensão e pesquisa ${ }^{1,10-12}$.

Então, ao se fazer a opção pela diversificação dos cenários de aprendizagem e inclusão de instituições públicas de saúde, as IES estão reconhecendo o importante papel dos serviços também como cenário de aprendizagem ${ }^{4,7,8}$. Assim, a valorização desses cenários deve provocar a ressignificação das práticas pedagógicas, especialmente, com adoção de metodologias ativas, centradas no estudante e com foco na comunidade. Para isto, é importante fortalecer a integração/ensino/serviço e comunidade, criando estratégias legais, de parceria, de trocas, de cooperação entre o sistema de educação e o de saúde $e^{10,13-16}$.

$\mathrm{Na}$ Odontologia, tradicionalmente, o ensino tem se baseado na transmissão de conhecimento e no desenvolvimento de habilidades psicomotoras, com foco nas doenças bucais e na clínica privada ${ }^{9}$. Entretanto, o processo de formação tem sofrido diversas mudanças. A partir da publicação das DCN para os cursos de Odontologia, e dos diversos dispositivos para reorientação da formação dos Ministérios da Saúde e da Educação, nos últimos anos, como o Programa Nacional de Reorientação da Formação Profissional em Saúde (PRO-SAÚDE), o Programa de Educação pelo Trabalho para a Saúde (PET-SAÚDE) e, mais recentemente, o Programa de Educação pelo Trabalho para a Saúde (PETGRADUASUS), as IES foram provocadas a mudar seus projetos pedagógicos diante dos novos desafios para a formação $0^{3,7,17}$.

A inserção de estudantes no mundo do trabalho oportuniza uma formação contextualizada, com base nas condições de saúde bucal da população, associada aos hábitos, 
valores e costumes de cada comunidade ${ }^{15,18-24}$. A experiência na comunidade, na formação em Odontologia, apresenta impacto positivo sobre os discentes, no que diz respeito a uma melhor compreensão das questões éticas e sociais relacionadas à saúde bucal das populações ${ }^{25-28}$; melhora o acesso aos serviços odontológicos; e aumenta a autonomia dos estudantes, além de aprimorar suas competências de gestão e habilidades técnicas ${ }^{26,29}$.

Em 2002, o curso de Odontologia da IES cenário desta pesquisa propôs e implementou um projeto pedagógico, reorientando a formação por meio da inserção de estudantes nos serviços públicos de saúde, desde os primeiros semestres/anos, e ao longo de todo o curso. Os estágios supervisionados passaram a ter carga horária semanal na matriz curricular do curso, em todos os semestres, seguindo uma lógica transversal e crescente de complexidade. Os primeiros estágios estão relacionados à atenção básica em unidades de Estratégia Saúde da Família (ESF), em seguida atenção secundária nos Centros de Especialidades Odontológicas (CEO) e atenção hospitalar.

Por outro lado, o município vem construindo, desde 2005, a Rede Escola/Sistema Saúde Escola, que é um dispositivo de organização, planejamento e avaliação para o fortalecimento da integração ensino/ serviço/comunidade. A Rede Escola/Sistema Saúde Escola também faz a gestão das pesquisas no município, organizando os fluxos e reorientando, na medida do possível, as pesquisas. Esse movimento tem provocado a reorganização da formação de estudantes e profissionais nos serviços de saúde, assim como a transformação das práticas que perfazem a gestão do cuidado na rede de atenção à saúde do município.

Dessa forma, este trabalho teve por objetivo compreender as percepções de estudantes de Odontologia de uma IES sobre os estágios supervisionados desenvolvidos na Rede de Atenção em Saúde Bucal de um município do Nordeste.

\section{MÉTODO}

$\mathrm{Na}$ busca de caminhos para responder ao objetivo da pesquisa, optou-se por uma trajetória estruturada em uma abordagem qualitativa. Este arcabouço teórico-metodológico oportuniza visualizar significações, impressões, valores e conceitos $^{30,31}$. Os estudantes, sujeitos dos estágios supervisionados, estão inseridos numa realidade social, pensam sobre o que fazem, aprendem e podem interpretar suas ações com base no vivido, produzido nos estágios supervisionados do curso de Odontologia, bem como da vivência partilhada com os outros estudantes, professores, usuários e preceptores nesse cenário ${ }^{30,32}$.

A pesquisa ocorreu nos meses de julho a setembro de 2011 em um curso de Odontologia de uma IES pública do Nordeste do Brasil. Foram convidados a participar os estudantes matriculados nos Estágios Supervisionados do curso, atendendo aos seguintes critérios de inclusão e exclusão: ter realizado o estágio supervisionado I do primeiro semestre do curso. Foram excluídos estudantes do primeiro período do curso, aqueles que estavam com interrupção da matrícula total ou parcial. A amostra foi do tipo intencional e por conveniência, a partir de lista fornecida pela Coordenação do Curso de Graduação.

Participaram da pesquisa 18 estudantes, sendo dois de cada semestre letivo, considerando que o curso de Odontologia tem 10 semestres. Para a construção do corpus da pesquisa, foram utilizadas entrevistas com roteiro semiestruturado. Compreende-se por entrevista uma conversa entre dois ou mais interlocutores com a finalidade de construção dialógica, a qual 
gera reflexão para coletar informações. O local e horário da realização das entrevistas foram previamente pactuados com os estudantes. A opção pela entrevista foi para que os participantes da pesquisa fossem convidados a falar de forma a aprofundar o diálogo, impressões, valores e significados sobre o tema estudado $^{30,31}$.

$\mathrm{O}$ roteiro semiestruturado continha perguntas que abordavam os seguintes temas: estágios e a formação acadêmica em Odontologia; estágios e a prestação dos serviços públicos de saúde no SUS; e avaliação de estratégias de mudanças para a melhoria das práticas desenvolvidas. Todas as entrevistas foram realizadas por um único pesquisador. Previamente às entrevistas, procedeu-se um treinamento teórico e prático sobre pesquisa qualitativa e entrevistas.

Antes do início das entrevistas, os participantes da pesquisa foram informados pelo pesquisador sobre o estudo, objetivos e procedimento da coleta e tratamento dos dados, assim como se deu a leitura do termo de consentimento livre e esclarecido (TCLE). As entrevistas foram gravadas em um gravador digital de voz e, posteriormente, transcritas pelo próprio entrevistador. Em média, duraram 30 minutos e, depois de transcritas, as gravações foram apagadas.

Em seguida, foi traçado o mapa horizontal do material. Assim, à luz do referencial teórico, bem como dos objetivos deste trabalho, foram realizadas leituras exaustivas e repetidas das transcrições, fazendo-se uma relação interrogativa com eles, a fim de apreender as estruturas relevantes, seguindo-se a análise de conteúdo ${ }^{33}$. Esse caminho permitiu elaborar uma classificação, por meio de uma leitura transversal. Posteriormente, a partir das estruturas de relevância, processou-se o enxugamento da classificação, reagrupando os temas mais relevantes para a análise final. Dessa forma, as categorias empíricas ficaram assim construídas: 1. Conhecimento sobre o SUS; 2. Potencialização do trabalho dos profissionais; 3. Diversificação dos cenários de aprendizagem.

A fim de assegurar o anonimato dos sujeitos da pesquisa, os estudantes foram codificados pela inicial "E" entre parênteses, acompanhada pelo numeral ordinal referente à ordem de realização das entrevistas: (E1), (E2), (E3) e assim sucessivamente.

A pesquisa foi aprovada pelo Comitê de Ética em Pesquisa com Seres Humanos sob o número 08/2011 da IES cenário da pesquisa. Os estudantes assinaram o TCLE, o qual continha informações sobre o estudo, sendo observados a garantia de anonimato, o sigilo e a autorização para divulgação dos dados coletados, conforme normas vigentes à época de realização do estudo.

\section{RESULTADOS E DISCUSSÃO}

A partir da análise realizada, identificaramse as seguintes categorias: conhecimento sobre o SUS; potencialização do trabalho dos profissionais; diversificação dos cenários de aprendizagem, as quais serão apresentadas a seguir.

\section{Conhecimento sobre o SUS}

$\mathrm{O}$ projeto pedagógico assumiu como compromisso a inserção gradativa dos estudantes na rede de serviços do SUS, conforme orientação das DCN. Dessa forma, reconhece-se o desafio de construir a Rede Escola/ Sistema Saúde Escola, compreendendo os cenários de atenção a Saúde como potentes para a formação em Odontologia. Este movimento acontece sempre na direção de uma experiência concreta com o SUS ${ }^{15,17-24}$.

Observou-se que os estudantes reconhecem que sua inserção nos estágios supervisionados oportuniza tanto conhecimento 
quanto vivências sobre o sistema de saúde em nível local.

"Os estágios supervisionados, eles são uma boa oportunidade para o estudante, tanto nos primeiros estágios, inserindo no serviço público, e ter conhecimento de como funciona toda rede pública dos serviços de saúde...”. (E12)

"A gente sempre consegue ter uma vivência, assim, de todas as etapas que são cumpridas na USF [Unidade Saúde da Família], desde os ACS [Agentes Comunitários de Saúde] até... até o fim, que a gente acompanha o dentista...". (E15)

Nesse sentido, participam do processo ensino-aprendizagem, nos serviços, outros atores importantes. Além de docentes e discentes, destacam-se os trabalhadores do SUS, quer sejam eles cirurgiões-dentistas ou não, incluindo-se, também, os de nível médio e, especialmente, os usuários e as comunidades/famílias.

A partir do planejamento coletivo, são respeitados os fluxos, as necessidades/demandas de cada território e de cada equipamento social. A execução das atividades respeita essas questões, como também o objetivo educacional de cada estágio supervisionado. O currículo foi implantado em 2002, e muitas mudanças aconteceram nos últimos anos. Muitos acertos, alinhamentos, discussões bem como muitas pactuações e oficinas foram realizados para ajustar as intenções entre a IES e a rede de serviços públicos odontológicos.

$\mathrm{Na}$ universidade, foi necessária a contratação de docentes com formação na área de saúde coletiva. Isso também ocorreu na área clínica, assim como na rede de atenção em saúde bucal, com a expansão tanto na atenção primária à saúde, orientada pela ESF, como na criação de CEO. Houve também a readequação dos cenários de aprendizagem, estivessem eles na própria instituição como também nos serviços de saúde bucal do município envolvido. Outro destaque importante foi a mobilização em torno da Rede Escola/Sistema Saúde Escola, a qual organiza os fluxos de estudantes na rede, como também dos projetos de extensão e pesquisa.

A IES participou de todos os editais de reorientação da formação de profissionais de nível superior. Nos estágios iniciais do curso, nos dois primeiros anos, há uma inserção do estudante na atenção básica com o objetivo de conhecer, observar, vivenciar e desenvolver ações de promoção de saúde e educação em saúde no território da ESF, na sala de espera, em visitas domiciliares ou envolvendo os equipamentos sociais da área adstrita, como escolas, creches, associação de moradores, igrejas e centros comunitários, entre outros.

Estudo desenvolvido por Almeida et al. $(2016)^{34}$ ressaltaram a importância da inserção de estudantes nos equipamentos sociais e do desenvolvimento de atividades de promoção de saúde. As atividades representaram a possibilidade de desenvolver competências de comunicação e linguagem ${ }^{3}$, como também de práticas mais horizontais na relação entre trabalhadores do SUS, estudantes e usuários, que aproximam a formação da realidade do trabalho $15,16,21,35$.

Outros estudos também enfatizam a importância de estágios extramuros para a melhoria de competências no que diz respeito à compreensão de questões relacionadas à diversidade cultural, ao cuidado ético do usuário e ao processo de adoecer das populações com vulnerabilidade social, sendo a formação em Odontologia com base na comunidade, uma estratégia apropriada para abordar essas questões $28,29,36$

No estudo de Noro e Torquato $(2010)^{16}$, os estudantes relataram aspectos positivos do SUS, embora se reconheceu a visão ainda 
preconceituosa de alguns atores da academia.

Os conhecimentos propostos para alcançar as competências também são ampliados, abordando-se questões de bioética, ética profissional, antropologia, sociologia e psicologia, associadas às relações interpessoais desses atores nos cenários de aprendizagem.

Os estudantes percebem a aproximação com o território e com a população.

“[...] os estágios supervisionados colocam a gente diretamente em contato com o público... e isso ajuda a gente a compreender como funciona o processo de conhecimento e obtenção do vínculo com a pessoa". (E1)

Os estudantes desenvolvem as atividades dos quatro estágios dos semestres iniciais no mesmo território. Assim, oportuniza-se a criação de vínculo com a equipe e com a comunidade, e, com a proposta de complexidade crescente de ações e atividades, podem planejar, executar e avaliar o vivenciado, problematizando o cotidiano. Em estágios supervisionados de semestres mais avançados, os estudantes executam a clínica odontológica na atenção básica, nesses territórios, associando as ações de promoção de saúde e educação em saúde, ou seja, com a rotina de trabalho da equipe de saúde bucal, seguindo a rotina de trabalho da ESF.

Os contatos entre estudantes, docentes, trabalhadores e a própria comunidade são importantes para a construção permanente de conhecimento e valorização das práticas. A aproximação com a comunidade pela problematização é uma oportunidade e um desafio para a aprendizagem significativa ${ }^{37,38}$.

Vale destacar que a vivência nos cenários de prática a partir dos estágios supervisionados com a discussão de temas como princípios do SUS e suas diretrizes, o processo histórico da luta para sua construção, desafios na organização do trabalho em saúde e a necessidade de empenho para sua consolidação oportunizam uma formação com capacidade crítica geradora de reflexões sobre desafios, avanços e fragilidades da política de saúde bucal no contexto do SUS ${ }^{15-}$ 23,35 .

Essas vivências procuram articular a teoria e a prática buscando a contextualização do trabalho da equipe de saúde bucal na atenção primária orientada pela ESF. Dessa forma, a reorientação da formação profissional visa à melhoria da qualidade da produção do cuidado em saúde bucal, na perspectiva do trabalho multiprofissional da equipe na ESF. Alguns estudos apontaram que os estudantes reconhecem os cenários de aprendizagem nos serviços públicos de saúde bucal como importantes para a formação em Odontologia ${ }^{22-24}$.

\section{Potencialização do trabalho dos profissionais}

$\mathrm{Na}$ articulação ensino/serviço, uma das potencialidades é que o estudante traz inovação ao trabalho em saúde. Isto desperta, no preceptor, a necessidade de educação permanente ${ }^{10,37}$. Assim, nesse movimento, os estudantes contribuem com criatividade e inovação e o preceptor com maturidade, conhecimento do território (epidemiológico, socioeconômico, cultural) e capacidade de articulação com os equipamentos sociais, visando às ações intersetoriais. Os estudantes reconhecem a importância da integração ensino/serviço, na perspectiva de ampliar as ações já desenvolvidas pelos trabalhadores.

"As atividades que a gente realizou foram de imensa ajuda para as unidades onde a gente estava. Todos os profissionais agradeceram bastante e a comunidade sempre se mostrou bastante satisfeita com todas as atividades que a gente fazia [...] sem falar da facilitação dos trabalhos das pessoas que já trabalham na unidade”. (E8) 
"Durante os estágios a gente chega a atender muitos pacientes, então..., $e$ atende em USF, né?, então isso contribui pra o SUS. E a gente coloca os nossos conhecimentos no atendimento." (E10)

$\mathrm{Na}$ perspectiva de mudanças na formação dos profissionais da saúde, está incluída a transformação da interface ensino/trabalho, ou seja, das relações entre o ensino e os serviços de saúde. Tais espaços ocupam agora lugar privilegiado na formação, ao desenvolverem, no estudante, a percepção acerca do outro no cotidiano do cuidado. Quando a integração ensino/serviço acontece de forma efetiva, a dicotomia entre o ensino e a produção dos cuidados em saúde é diminuída. Assim, não é possível pensar a mudança na formação dos profissionais de saúde sem a discussão sobre a integração ensino/serviço ${ }^{10-16}$.

Bulgarelli et $a .^{35}$ ao investigarem a percepção de estudantes sobre os estágios na atenção primária, observaram uma mudança gradual e positiva na rotina do serviço, bem como os estudantes relataram ressignificação da percepção sobre os serviços, além de mencionarem o impacto dos estágios em si mesmos. O estágio propiciou uma mudança de olhar sobre a prática, favorecendo a realização de um número maior de atividades clínicas no serviço e de procedimento coletivos em Odontologia. As atividades coletivas são as desenvolvidas no campo da promoção da saúde e educação em saúde bucal na perspectiva freiriana $^{38}$. O perfil epidemiológico, social e cultural do território e seus equipamentos sociais direcionam as temáticas a serem abordadas em cada semestre letivo.

A reflexão gerada ao final das atividades ressignifica a própria prática, com o envolvimento de docentes, estudantes e preceptores $^{39}$. As atividades são registradas em relatos mensais e resenhas de textos que sustentam a prática. A avaliação é processual e realizada por meio de portfólio nos estágios iniciais; já nos estágios dos últimos anos do curso, a opção foi pelo diário de campo.

A integração ensino/serviço/comunidade configura-se como complexa, envolvendo interesses, saberes e poderes. Convergir agendas do setor saúde e educação é um desafio permanente. Assim, do ponto de vista legal, existe convênio entre as duas instituições para o desenvolvimento dos estágios. Há um compromisso assumido pela IES e pelo serviço na promoção da integração ensino/serviço visando à melhoria da qualidade da atenção à saúde e propiciando uma formação voltada à consolidação do SUS.

Diante dessa compreensão, o diálogo também é construído onde o estágio acontece. Diante dos objetivos de aprendizagem dos estágios e das demandas do território e da equipe ESF, as ações são planejadas, executadas e avaliadas. O objetivo é o desenvolvimento de competências, habilidades e atitudes para o cirurgião-dentista, considerando o trabalho desse profissional no SUS. À medida que o estudante é inserido nos serviços públicos de saúde, é importante que suas ações e atividades possam potencializar, ampliar as que já são executadas pelos serviços, com vistas à integralidade e à humanização do cuidado em saúde bucal ${ }^{35,39}$.

O fazer/aprender/fazer foi e ainda é novo para todos. À medida que a universidade se aproxima dos serviços, fazendo e aprendendo ao mesmo tempo e junto, há um aumento na compreensão de que o aprender vem da realidade, e que essa produção de conhecimento aproxima, sendo capaz de gerar reflexões sobre os modos de trabalho do docente, da equipe e do estudante. Este modo de agir vem sendo desafiador, pois traz uma nova característica às salas de aula intramuros: a construção coletiva, de forma dialógica e participativa, com e para os 
serviços de saúde, a partir das condições de trabalho ${ }^{7,27-29}$.

Portanto, são necessárias mudanças profundas nas concepções, nas práticas e nas relações de poder das universidades, nos serviços de saúde e no campo das políticas. Além disso, é necessária a criação de espaços democráticos para a construção ampliada e compartilhada dos sujeitos envolvidos, para uma reflexão crítica e plural, com vistas à construção de canais de comunicação e discussão coletiva, visando à formação profissional ${ }^{29}$. Afinal, no território, diversas são as expressões de poder, diferentes são as identidades; é, pois, importante o reconhecimento das diferenças e das identidades.

\section{Diversificação dos cenários de aprendizagem}

As DCN estimulam a diversificação dos cenários de aprendizagem, vislumbrando o desenvolvimento de competências: tomada de decisão, gestão de serviços públicos de saúde, liderança e administração, e educação permanente $^{40}$. Diante do já exposto, os estágios supervisionados na atenção primária, secundária e terciária, como também na gestão do SUS, são importantes cenários da formação profissional.

$O$ entendimento de que a realidade deve ser o objeto do aprendizado, considerando o previsto, o imprevisto, invenção e criatividade para possibilidades diante do desconhecido, a diversificação de cenários de aprendizagem conduz às mudanças das práticas e da formação em saúde, induzindo novas formas de aprender/ensinar em Odontologia ${ }^{8}$.

Em seus depoimentos, os estudantes também ressaltaram a inserção gradual de ações de complexidade, no SUS, conforme destacaram as $\mathrm{DCN}^{6}$.

"A gente começa acompanhando os ACS, fazendo visitas domiciliares [...] $e$, depois, a gente vai progredindo, até chegar no cirurgião-dentista, que a gente vê a vivência dele, atuando assim [...] não só na USF, como nas outras coisas: na creche, nas escolas. Nas instituições que têm pros idosos, também, a gente fez...". (E15)

As demandas da academia e da gestão dos serviços de saúde nem sempre são compatíveis e, para tanto, são necessários pactuações, ajustes e modificações ${ }^{14}$. Essas pactuações devem ser realizadas com o objetivo de desenvolver ações conjuntas e articuladas para a contribuição recíproca na proposta de formação de trabalhadores/ profissionais de saúde, no sentido de elaborar e construir conhecimentos, e de produzir cuidado em saúde qualificado e resolutivo ${ }^{6,41}$.

É importante destacar que uma matriz curricular nova é fruto de muita discussão entre docentes, gestão acadêmica, discentes, lideranças, técnicos administrativos e gestão de serviços. Desta forma, a inserção de estudantes gera expectativas não só nos próprios estudantes, como também nos docentes e trabalhadores do SUS envolvidos. As vivências novas e inexploradas proporcionam a construção do sujeito social em todos os envolvidos no processo. $\mathrm{O}$ aprendizado acumulado com base nas experiências que deram certo e errado, nos caminhos trilhados e no olhar aguçado de docentes e trabalhadores pode fomentar mudanças ${ }^{35}$.

“... os estágios do começo do curso, aqueles que a gente vai na comunidade, aquela questão né, eu acho que eles, assim, contribuem muito mais pra formação da gente do que esses estágios do final que a gente vai lá no CEO, na USF pra atender. Eu não sei se por uma dificuldade das próprias pessoas que tão lá orientando a gente e que não deixam a gente fazer nada...ou então, não sei, a própria condição da unidade lá que não 
tem material, não tem...então acaba que a gente acaba fazendo assim " (E10)

As pactuações e o diálogo são pontos de partida para o entendimento do que se deseja para a formação e do que o território necessita. Práticas dialógicas e participativas entre docentes, trabalhadores do SUS e gestão devem ser instituídas. Assim, pode-se pensar em formação de profissionais para produzir ações e serviços de saúde com vistas à equidade, à resolutividade e à integralidade no SUS. Nessa perspectiva, os cursos não devem predefinir propostas de trabalho, mas adequar as atividades demandadas pelos serviços e necessidades da população aos objetivos educacionais da formação profissional ${ }^{14}$.

A diversificação dos cenários de aprendizagem compreende que os serviços prestadores de atenção em saúde também são locais onde se aprende. Os estudantes se veem como trabalhadores/protagonistas/sujeitos, apropriando-se de um novo fazer/saber em saúde, com base na responsabilização, na autonomia e no respeito ao outro ${ }^{3,4}$. Destacam, assim, a necessidade de vivenciar cenários de estágios em toda a rede de serviços de saúde bucal, inclusive, no âmbito da gestão dos serviços, o que tem despertado diversas discussões.

“... eu acho importante, não só se deter na unidade de saúde da família, mas também em hospitais e centros especializados". (E5)

"Uma sugestão que eu teria é você poder atuar em vários períodos, ao longo do curso. Tipo: você ter vários campos de atuação, ou seja, você não ficar só na USF...”. (E12)

Os avanços orientados pelas DCN apontam para mudanças na formação profissional que contemplem elementos de fundamentação essencial no seu campo do saber ou profissão, em uma concepção de autonomia do estudante e do profissional de saúde, engajados em um processo de educação permanente. Para tanto, os cursos de graduação devem ter como base a aprendizagem ativa, centrada no aluno como sujeito da aprendizagem e no professor como facilitador e mediador desse processo ensino-aprendizagem.

A partir deste estudo e considerando que o cenário foi um curso de Odontologia, com estudantes, é salutar destacar que os achados retratam uma realidade singular, que pode divergir de outros cenários e sujeitos. Sugere-se a realização de novas pesquisas buscando o diálogo com outros interlocutores no cenário de ensino, serviço e comunidade.

Por outro lado, os resultados geram reflexões e contribuições importantes, as quais subsidiam a inserção de estudantes nos cenários de aprendizagem em serviços públicos de saúde. Também contribuem para a gestão dos serviços de saúde e para a IES na perspectiva do fortalecimento do SUS, visando à formação de profissionais comprometidos com a qualidade da produção do cuidado em saúde.

\section{CONCLUSÕES}

Os estudantes de Odontologia compreendem a importância dos estágios supervisionados para a sua formação acadêmica, uma vez que estes permitem mais aproximação com a população, conhecimento e vivência do SUS, experiência profissional, desenvolvimento humanista e atuação extramuros em cenários diversificados de prática.

$\mathrm{Na}$ percepção dos estudantes, os estágios contribuem com o desenvolvimento de novas estratégias para o serviço, a educação permanente em saúde, a realização de atividades de promoção, prevenção e educação em saúde, disparam processos de participação popular e potencializam o trabalho dos profissionais do serviço. Com isso, a formação volta-se para o SUS, que será novamente beneficiado com a 
inserção desses futuros profissionais na rede de serviços.

As sugestões e estratégias destacadas pelos estudantes para a melhoria das práticas desenvolvidas nos estágios supervisionados estão relacionadas a uma maior preocupação com o desenvolvimento de competências e habilidades necessárias durante os estágios. Destarte, a formação contribui para fortalecimento do SUS, visando a integralidade do cuidado e ao acesso universal e de qualidade aos serviços públicos de saúde.

\section{ABSTRACT \\ Dentistry training and supervised internship in public health services: students' view and experiences}

The aim of this study was to understand the perceptions of Dental students from a public university about the supervised internships in public health services. It is a qualitative research with the use of semi-structured interviews with 18 students, selected for convenience, enrolled from the first to the last year of the course. The interviews, audiotaped and transcribed, were analyzed using the content analysis technique. The proximity with the population, the humanized professional experience and the knowledge built by the experience in the services were highlighted, producing new strategies of action in the Brazilian Health System (SUS). The suggestions were related to the diversification of practice scenarios and better planning of activities for the development of skills and abilities. Through the internships, the training turns to the SUS, which will again benefit from the inclusion of these future professionals in the service network, with consequent improvement in the population's health care.

Descriptors: Health Education. Teaching Care Integration Services. Education, Dental.

\section{REFERÊNCIAS}

1. Werneck MAF, Senna MIB, Drumond MM, Lucas SD. Nem tudo é estágio: contribuições para o debate. Ciênc Saúde Colet. 2010;15(1):221-31.

2. Ceccim RB, Ferla AA. Educação e saúde: ensino e cidadania como travessia de fronteiras. Trab Educ Saúde. 2009; 6(3): 443-56.

3. Vendruscolo C, Padro, ML, Kleba ME. Integração ensino-serviço no âmbito do Programa Nacional de Reorientação da Formação Profissional em Saúde. Ciênc Saúde Colet. 2016; 21(9): 2949-60.

4. Haddad AE, Laganá DC, Assis EQ, Morita $\mathrm{MC}$, Toledo AO, Rode SM et al. A aderência dos cursos de graduação em odontologia às Diretrizes Curriculares Nacionais. In: Ministério da Saúde (BR); Ministério da Educação (BR). A aderência dos cursos de graduação em Enfermagem, Medicina e Odontologia às diretrizes curriculares nacionais. Brasília, DF: Ministério da Saúde; 2006. p. 119-52. (Série F, Comunicação e Educação na Saúde).

5. Brasil. Lei n. 9.394, de 20 de dezembro de 1996. Diretrizes e Bases da Educação Nacional. Educação profissional: legislação básica. Brasília, DF: PROEP, 1998.

6. Brasil. Ministério da Educação. Conselho Nacional de Educação. Câmara de Educação Superior. Diretrizes Curriculares Nacionais do Curso de Graduação em Odontologia. Diário Oficial da União. 2002 mar. 4; Seção 1, p. 10

7. Silva MAM, Amaral JHL, Senna MIB, Ferreira EF. The National Reorientation of Vocational Healthcare Training (Pro-Saúde) and encouragement of inclusion of differentiated learning scenarios within Dentistry courses in Brazil. Interface (Botucatu). 2012; 16(42): 707-17.

8. Ferreira MLSM, Cotta RMM, Oliveira MS. Reconstrução teórica do cuidado para as práticas de saúde: um olhar a partir da 
produção de alunos de curso de especialização a distância. Rev Bras Educ Méd. 2008; 32(3):291-300.

9. Zilbovicius C, Araujo ME, Botazzo C, Frias AC, Junqueira SR, Junqueira CR. A paradigm shift in predoctoral dental curricula in Brazil: evaluating the process of change. J Dent Educ. 2011; 75(4): 557-64.

10. Aguiar Neta A, Alves MSCF. Comunidade como local de protagonismo na integração ensino-serviço e atuação multiprofissional. Trab Educ Saúde. 2016; 14(1): 221-35.

11. Carvalho SOB, Duarte LR, Guerrero JMA. Parceria ensino e serviço em unidade básica de saúde como cenário de ensinoaprendizagem. Trab Educ Saúde. 2015; 13(1): 123-44

12. Ribeiro IL, Medeiros Júnior A. Undergraduate education in health, a reflection on teaching-learning. Trab. educ. saúde. 2016; 14(1): 33-53.

13. Forte FDS, Ribeiro TR, Freitas CHSM, Barbosa AS, Morais MB, Braga CC, Filgueiras F. Community-based education: experience in community health supervised clerkships at the Federal University of Paraíba Dentistry Course. In: Bolela V, Germani AC, Holanda H, Amaral E. Community-based education for the health professions: learning from the Brazilian experience. 1ed. Whashington: OPAS, 2015, v.1, p. 220-32.

14. Toassi RFC, Baumgarten A,Warmling CM, Rossoni E, Rosa AR, Slavutzk SMB. O ensino nos serviços de atenção primária do Sistema Único de Saúde na formação de profissionais de saúde no Brasil. Interface (Botucatu). 2013; 17(45): 385-92

15. Finkler M, Caetano JC, Ramos FRS. Integração "ensino-serviço" no processo de mudança na formação profissional em Odontologia. Interface (Botucatu). 2011; 15(39): 1053-67.
16. Noro LRA, Torquato SM. Percepção sobre o aprendizado de saúde coletiva e o SUS entre alunos concludentes de curso de odontologia. Trab Educ Saúde. 2011; 8(3): 439-47.

17. Vendruscolo C, Ferraz F, Prado ML, Kleba ME, Reibnitz KS. Teaching-service integration and its interface in the context of reorienting health education. Interface (Botucatu). 2016; 20(59): 1015-25.

18. Warmiling CM, Rossoni E, Hugo FN, Toassi RFC, Lemos VA, Slavutzki SMB, et al. Estágios curriculares no SUS: experiências da Faculdade de Odontologia da UFRGS. Rev ABENO. 2011; 11(2): 63-70.

19. Souza Nétto OB, Moura MS, Lima MDM, Lages GP, Mendes RG, Moura LFAD. O Pró-Saúde no curso de Odontologia na Universidade Federal do Piauí: relato de uma vivência de cinco anos. Ciênc Cuid Saúde. 2013; 12(2): 391-7.

20. Alves LA, Freires IA, Braga CC, Castro RD. Experiência exitosa na atenção odontológica à comunidade. Rev Bras Ciênc Saúde. 2012; 16(2):235-8.

21. Albuquerque VS, Gomes AP, Rezende AHA, Sampaio MX, Dias OV, Lugarinho RM. A. integração ensino-serviço no contexto dos processos de mudança na formação superior dos profissionais da Saúde. Rev Bras Educ Méd. 2008; 32(3): 356-62.

22. Morita MC, Kriger L, Carvalho ACP, Haddad AE. Implantação das Diretrizes Curriculares Nacionais em Odontologia. 2a ed. Maringá: Dental Press, Abeno, OPS, MS; 2013.

23. Almeida AB, Alves MS, Leite ICG. Reflexões sobre os desafios da odontologia no Sistema Único de Saúde. Rev APS. 2010; 13(1): 126-32. 
24. Silveira JLGC, Garcia VL. Mudança curricular em Odontologia: significados a partir dos sujeitos da aprendizagem. Interface (Botucatu) 2015; 19 (52): 145-58.

25. Taichman RS, Parkinson JW, Nelson BA, Nordquist B, Thompson JF. Leadership training for oral health professionals: a call to action. J Dent Educ. 2012; 76(2): 185-91.

26. Aston-Brown RE, Branson B, GadburyAmyot CC, Bray KK. Utilizing public health clinics for service-learning rotations in dental hygiene: a four-year retrospective study. J Dent Educ. 2009; 73(3): 358-74

27. Bean CY. Community-based dental education at the Ohio State University: The OHIO Project. J Dent Educ. 2011; 75 (Supp 1): $25-35$.

28. Hood JG. Service-learning in Dental Education: Meeting Needs and challenges. J Dent Educ. 2009; 73(4): 454-463.

29. Mascarenhas AK. Community-Based Dental Education at Boston University. J Dent Educ. 2011; 75(supp 1): 21-24.

30. Minayo MCS. O desafio do conhecimento: pesquisa: pesquisa qualitativa em saúde. São Paulo: Hucitec, 2015. 406 p.

31. Deslandes S, Gomes R, Minayo MCS (org). Pesquisa Social: teoria, método e criatividade. $33^{\mathrm{a}}$ ed. Petrópolis: Vozes, 2013.

32. Hanguette TMF. Metodologias qualitativas na sociologia. Petrópolis: Vozes; 2013.

33. Bardin L. Análise de Conteúdo. Lisboa: Edições 70; 2014. 280 p.

34. Almeida PF, Bastos MO, Condé MA, Macedo NJ, Feteira JM, Botelho FP, Silva RL. Trabalho de Campo Supervisionado II: uma experiência curricular de inserção na Atenção Primária à Saúde. Interface (Botucatu). 2016; 20(58): 777-86.

35. Bulgarelli AF, Souza KR, Baumgarten A, Souza JM, Rosing CR, Toassi RFC. Formação em saúde com vivência no
Sistema Único de Saúde (SUS): percepções de estudantes do curso de Odontologia da Universidade Federal do Rio Grande do Sul (UFRGS), Brasil. Interface (Botucatu). 2014; 18(49): 351-62.

36. Mashabi S, Mascarenhas AK. Impact of community externships on the clinical performance of senior dental students. J Dent Educ. 2011; 75(10 Suppl): S36-S41.

37. Palmier AC. Inserção do aluno de Odontologia no SUS: contribuições do PróSaúde. Rev Bras Educ Méd. 2012; 36 (Supl. 2): 152-7.

38. Cyrino EG, Toralles-Pereira ML. Trabalhando com estratégias de ensinoaprendizado por descoberta na área da saúde: a problematização e a aprendizagem baseada em problemas. Cad Saúde Pública. 2004; 20(3): 780-788.

39. Forte FDS, Forte FDS, Pessoa TRRF, Freitas CHSM, Pereira CAL, Carvalho Junior PM. Reorienting dental education: the preceptor's view of supervised internship in the Brazilian Health System (SUS). Interface (Botucatu). 2015; 19 Supl 1: 83143.

40. Fonseca EP. As diretrizes curriculares nacionais e a formação do cirurgião dentista brasileiro. J Manag Prim Health Care. 2012; 3(2): 158-78.

41. Feuerwerker L. Além do discurso de mudança na educação médica: processos e resultados. São Paulo: Hucitec, 2002.

\section{Correspondência para:}

Franklin Delano Soares Forte e-mail: franklin@ccs.ufpb.br Universidade Federal da Paraíba

Centro de Ciências da Saúde Departamento de Clínica e Odontologia Social Campus Universitário I 58051-900 João Pessoa/PB 